\title{
Entwicklung einer Methode zur Performance- bewertung ausgewählter Lagerbereiche im Kontext des Lean Warehousing
}

\author{
Development of a Method for Performance Evaluation of Selected Warehouse \\ Areas in the Context of Lean Warehousing
}

\author{
Nina Vojdani \\ Mathias Knop \\ Lehrstuhl Produktionsorganisation und Logistik \\ Fakultät für Maschinenbau und Schiffstechnik \\ Universität Rostock
}

D er Beitrag stellt eine Methode zur Performancebewertung ausgewählter Bereiche eines Distributionszentrums im Kontext des Lean Warehousing vor. Auf Grundlage der Kundenanforderungen erfolgt die Entwicklung eines Kennzahlensystems sowie die Vorstellung und Diskussion zweier Möglichkeiten der Kennzahlenaggregation, die die Berücksichtigung unscharfer Daten in die Performancebewertung ermöglichen. Anhand eines Fallbeispiels wird die Methode veranschaulicht.

[Schlüsselwörter: Performancebewertung, Distributionszentrum, Lean Warehousing, Fuzzy-Logik]

$\mathbf{T}$ his paper presents a method for performance evaluation of selected areas of a distribution center in the context of lean warehousing. Based on customer requirements a performance evaluation system is developed. Two opportunities to aggregate performance metrics are presented and discussed. The aggregation approaches allow a consideration of fuzzy data in performance evaluation. A case study is used to illustrate the performance evaluation methods.

[Keywords: performance evaluation, distribution center, lean warehousing, fuzzy logic]

\section{EINFÜHRUNG}

Ein verschärfter Wettbewerb, ein turbulentes Marktumfeld und eine hohe Wechselbereitschaft der Kunden konfrontieren Unternehmen permanent mit der Notwendigkeit, vorhandene Logistiksysteme und Distributionsstrukturen im Hinblick auf ihre Performance zu prüfen, um notwendige Modifikationen im Bedarfsfall anzustoßen. Die zielgerichtete Planung und der Betrieb effizienter Distributionssysteme orientieren sich an den Anforderungen der Kunden sowie daraus abgeleiteten Logistikzielen. Distributionszentren - als wesentliche Bestandteile von
Distributionssystemen - leisten bei der Erfüllung der Kundenanforderungen einen entscheidenden Beitrag. Aktuell wird unter der Bezeichnung ,Lean Warehousing“ die Vision diskutiert, Distributionszentren bzw. Lager verschwendungsarm auszurichten. Neben dieser Vision werden in der Literatur zahlreiche weitere Ziele, wie zum Beispiel hohe Lieferflexibilität, -qualität und -bereitschaft, thematisiert [SG96; Wis09].

Ziel dieses Beitrags ist es, eine Methode zur Performancebewertung ausgewählter Bereiche eines Distributionszentrums vorzustellen. Ausgehend von Kundenanforderungen wird eine Leistungsbewertung vorgenommen. Am Beispiel eines Unternehmens aus dem Lebensmitteleinzelhandel wird die Methode zur Performancebewertung veranschaulicht. Der Beitrag fokussiert eine Performancebewertung anhand von Lean-Warehousing-Zielen.

Die Grundlage der Performancebewertung bilden die Konzeption eines Kennzahlensystems sowie der Einsatz der Fuzzy-Logik. Im ersten Schritt wird ein Kennzahlensystem erarbeitet, das bereichsspezifisch performancebezogene Kennzahlen systematisiert. Die in diesem Beitrag vorgestellte Methodik erlaubt eine Adaption an lagerspezifische Ziele und Abläufe unter Berücksichtigung der Lean-Warehousing-Philosophie. Im zweiten Schritt werden die Kennzahlen zur Performancebewertung unter $\mathrm{Zu}$ hilfenahme der Fuzzy-Logik aggregiert. Zur Modellierung der Kennzahlen und einer ggf. erforderlichen Gewichtung werden linguistische Terme verwendet. Die Anwendung der Fuzzy-Logik ermöglicht eine Berücksichtigung nicht oder nur unzureichend quantifizierbarer Ziele (z. B. schlanke oder flexible Lagergestaltung), unscharfer Kennzahlenwerte und Gewichtungsfaktoren bei der Performancebewertung.

Die entwickelte Methode kann Planer und Betreiber von Distributionszentren bei der Performancebestimmung ausgewählter Lagerbereiche unterstützen. 


\section{BEGRIFFLICHE GRUNDLAGEN}

\subsection{LOGISTIK-PERFORMANCE}

Der Begriff Performance bzw. Leistung wird in der Literatur unterschiedlich definiert [Hel08; Stö02]. Die Logistikprozessleistung kann nach Kühner als mengenund qualitätsbezogenes Ergebnis von Logistikaktivitäten in Relation $\mathrm{zu}$ den eingesetzten Ressourcen verstanden werden [Küh05]. Diese Output-Input-Relation ist am operativen Leistungsprozess der Logistik orientiert. Eine Ausrichtung an strategischen Aspekten des Logistikerfolgs erfordert darüber hinaus eine Integration von Aspekten der Zukunftssicherung sowie der Kundenzufriedenheit [Vas08]. Die Berücksichtigung aller Aktivitäten am Endverbrauchernutzen rückt damit in den Fokus der Performancebewertung. Stölzle konstatiert, dass ein maximaler Lieferservice nicht mit einem maximalen Kundennutzen einhergehen muss, vielmehr muss sich die Performancebewertung an den Anforderungen der Kunden an die Logistikprozesse orientieren [Stö02]. Stölzle schlägt eine Orientierung am Wertbeitrag vor [Stö02]. Diesem Beitrag liegt nachfolgendes Verständnis über die Logistikperformance zugrunde: Logistikperformance charakterisiert den Wertbeitrag logistischer Aktivitäten unter Berücksichtigung der Kundenanforderungen. Der Wertbeitrag logistischer Aktivitäten ist das Ergebnis der Zielerreichung.

Dieses Performanceverständnis erfordert eine an den Anforderungen der Kunden oder anderer relevanter Stakeholder orientierte Entwicklung von Logistikzielen für ein Distributionszentrum. Alicke et al. konstatieren, dass der Betrieb von Distributionszentren in der Praxis häufig nicht an den Anforderungen der Kunden orientiert ist [ALMS08]. Stölzle und Bachmann sehen weiteren Forschungsbedarf in Bezug auf ein ziel- bzw. strategieorientiertes und prozessorientiertes Performancemanagement [SB08].

\subsection{LEAN WAREHOUSING}

Bezugsrahmen dieses Beitrags bildet das Lean Warehousing. Lean Warehousing bezeichnet die Übertragung bzw. Adaption von Lean-Management-Prinzipien und Methoden auf die Lagerlogistik. Lean Warehousing ist durch eine kontinuierliche Wert-, Ressourcen- und Performanceorientierung charakterisiert [Chu09].

Wertorientierung bezeichnet dabei die Gestaltung, den Betrieb und die Wahrnehmung kontinuierlicher Verbesserungen anhand der Vision des Unternehmens und zugehöriger Distributionszentren. Ziel ist es in diesem $\mathrm{Zu}-$ sammenhang, geteilte Werte zu schaffen, die von allen Mitarbeitern verinnerlicht und gelebt werden. Relevante Werte leiten sich aus Kunden-, Mitarbeiter sowie Shareholderanforderungen $\mathrm{ab}$.

Ressourcenorientierung im Lean Warehousing bezeichnet den effizienten Ressourceneinsatz und damit das
Vermeiden und Reduzieren von Verschwendung und Inflexibilität. Methoden wie die Wertstromanalyse, Activity Based Costing oder Line Balancing tragen der Bedeutung der Ressourcenorientierung Rechnung. Ziel ist es, größeren Wert mit geringerem Mitteleinsatz zu erbringen. Womack und Jones verstehen unter Verschwendung jede menschliche Aktivität, die Ressourcen absorbiert, aber keinen Wert generiert [WJ03]. Goldsby und Martichenko schlagen für den Bereich der Logistik die Unterscheidung folgender Quellen der Verschwendung vor: Bestände, Transporte, Flächen, Zeit, Verpackung, Administration und Wissen [GM05].

Performanceorientierung bezeichnet die ergebnisbezogene Messung erzielter Leistungen innerhalb eines Lagers. Die Kommunikation der Leistungen unter den Mitarbeitern des Distributionszentrums ist von besonderer Bedeutung. Die Schaffung transparenter Prozesse soll eine Fehlervermeidung unterstützen und kontinuierliche Verbesserungsprozesse anstoßen. Ein Performancemanagement hat sich in Distributionszentren kaum etablieren können, Daten werden häufig nicht mit dem Ziel einer Performanceverbesserung aufgenommen [ALMS08].

In der Literatur werden darüber hinaus vielfältige Ansätze für das Lean Warehousing diskutiert: Verringerung von Behältergrößen, Lagerung von Schnelldrehern in der Nähe des E/A-Punkts, Standardbehälter und Kommissionierwege für jeden Lagerartikel, Einteilung des Arbeitstags bzw. der Arbeitsaufgaben in Standardarbeitstakte, Synchronisieren von Kommissionierung, Verpackung und Versand, kontrollierter Arbeitsfortschritt und Prozessverbesserung [JHR97]. Einen Überblick über wichtige Lean-Warehousing-Methoden gibt Abbildung 1.

\section{STAND DER Forschung ZUR PERFORMANCE- BEWERTUNG VON DISTRIBUTIONSZENTREN}

Zur Performancebewertung eines Distributionszentrums können verschiedene Methoden eingesetzt werden, z. B. analytische Verfahren, Simulation oder Methoden, die die Berücksichtigung von unsicheren oder qualitativen Daten erlauben. Der Einsatz analytischer Methoden der Performance- bzw. Leistungsbewertung ist in der Literatur eingehend untersucht, z. B. für ausgewählte Bereiche wie die Lagerung [Lee97; See06; Gla08]. Anzumerken ist, dass diese Methoden vorrangig zur Bewertung quantitativer Zielgrößen herangezogen werden können. Qualitativ geprägte Ziele, wie z. B. hohe Flexibilität, können nur unzureichend durch analytische Modelle charakterisiert werden. Die Simulation ist häufig mit hohem Aufwand verbunden. Die Abbildung qualitativer Ziele gestaltet sich ebenfalls schwierig. Des Weiteren sind Kenntnisse über zugrunde gelegte Wahrscheinlichkeitsverteilungen erforderlich. Lin konstatiert, dass die Performancebewertung in Distributionszentren durch zahlreiche unsichere Faktoren geprägt ist [Lin10]. Die Nutzung von Verfahren, die die Berücksichtigung von unsicheren oder qualitativen Daten 
erlauben, bietet Möglichkeiten, eine Performancebewertung auch im Hinblick auf qualitative Ziele vorzunehmen.
Einen möglichen Modellierungsansatz bietet die FuzzyLogik [Zah65], die in diesem Beitrag Verwendung findet.

\begin{tabular}{|c|c|c|c|c|c|c|c|c|c|}
\hline \multirow{2}{*}{$\begin{array}{c}\text { Construct } \\
1 . \\
\begin{array}{c}\text { Standardized } \\
\text { Processes }\end{array}\end{array}$} & \multicolumn{9}{|c|}{ Lean Practice } \\
\hline & SOPs & $\begin{array}{c}\text { Standardized } \\
\text { Work/Planning }\end{array}$ & $\begin{array}{l}\text { Commodity } \\
\text { Grouping }\end{array}$ & $\begin{array}{c}\text { Common } \\
\text { Processes \& } \\
\text { Best Practices }\end{array}$ & $\begin{array}{c}\text { Trailer } \\
\text { Loading \& } \\
\text { Unloading }\end{array}$ & $\begin{array}{c}\text { Routing \& } \\
\text { Travel Paths }\end{array}$ & - & - & - \\
\hline 2. People & $\begin{array}{c}\text { Safety \& } \\
\text { Ergonomics }\end{array}$ & $\begin{array}{c}\text { Leadership } \\
\text { Direction/Roles }\end{array}$ & $\begin{array}{l}\text { Management } \\
\text { Style }\end{array}$ & $\begin{array}{l}\text { Cross- } \\
\text { Training }\end{array}$ & $\begin{array}{c}\text { Teamwork \& } \\
\text { Empowerment }\end{array}$ & $\begin{array}{c}\text { Power } \\
\text { Distance \& } \\
\text { Daily } \\
\text { Involvement }\end{array}$ & $\begin{array}{l}\text { Recognition \& } \\
\text { Compensation }\end{array}$ & $\begin{array}{c}\text { Communication } \\
\text { Strategy }\end{array}$ & $\begin{array}{l}\text { Absenteeism } \\
\text { \& Turnover }\end{array}$ \\
\hline $\begin{array}{l}\text { 3. Quality } \\
\text { Assurance }\end{array}$ & $\begin{array}{c}5 \text { Whys, Root } \\
\text { Cause \& } \\
\text { Pareto }\end{array}$ & $\begin{array}{l}\text { Inspection \& } \\
\text { Autonomation }\end{array}$ & $\begin{array}{l}\text { Error Proofing } \\
\text { Methodology }\end{array}$ & $\begin{array}{l}\text { Inventory } \\
\text { Integrity }\end{array}$ & $\begin{array}{c}\text { Product \& } \\
\text { Process } \\
\text { Quality }\end{array}$ & $\begin{array}{l}\text { Quality } \\
\text { Metrics }\end{array}$ & - & - & - \\
\hline $\begin{array}{l}\text { 4. Visual } \\
\text { Management }\end{array}$ & $\begin{array}{l}\text { Value Stream } \\
\text { Mapping }\end{array}$ & $\begin{array}{l}\text { Process Control } \\
\text { Boards }\end{array}$ & $\begin{array}{c}\text { Metrics \& KPI } \\
\text { Boards }\end{array}$ & $\begin{array}{c}\text { Lean } \\
\text { Tracking }\end{array}$ & $\begin{array}{c}\text { Visual } \\
\text { Controls }\end{array}$ & $\begin{array}{l}\text { Andon } \\
\text { Systems }\end{array}$ & $\begin{array}{l}\text { (A3) One Page } \\
\text { Reports }\end{array}$ & - & - \\
\hline $\begin{array}{l}\text { 5. Workplace } \\
\text { Organization }\end{array}$ & $5 \mathrm{~S}$ & $\begin{array}{c}\text { Signage \& } \\
\text { Shadow Boards }\end{array}$ & Cleanliness & $\begin{array}{c}\text { Supply \& } \\
\text { Material } \\
\text { MGMT }\end{array}$ & $\begin{array}{l}\text { Point of Use } \\
\text { Storage }\end{array}$ & $\begin{array}{l}\text { ID Problem } \\
\text { Parts Areas }\end{array}$ & - & - & - \\
\hline 6. Lot Sizing & Batch Sizes & WIP & $\begin{array}{l}\text { Kanban } \\
\text { Systems }\end{array}$ & $\begin{array}{c}\text { Quick } \\
\text { Changeover }\end{array}$ & $\begin{array}{c}\text { Lead Time } \\
\text { Tracking }\end{array}$ & $\begin{array}{l}\text { Inventory } \\
\text { Turns }\end{array}$ & $\begin{array}{c}\text { Order } \\
\text { Frequency }\end{array}$ & - & - \\
\hline $\begin{array}{l}\text { 7. Material } \\
\text { Flow }\end{array}$ & Pull Systems & $\begin{array}{l}\text { Leveled Flow \& } \\
\text { Work }\end{array}$ & FIFO & $\begin{array}{l}\text { Layout \& } \\
\text { Zones }\end{array}$ & $\begin{array}{l}\text { Velocity \& } \\
\text { Slotting }\end{array}$ & $\begin{array}{c}\text { Travel } \\
\text { Distance }\end{array}$ & $\begin{array}{l}\text { Cellular } \\
\text { Structure }\end{array}$ & $\begin{array}{l}\text { Demand } \\
\text { Stabilization }\end{array}$ & Cross-Docking \\
\hline $\begin{array}{l}\text { 8. Continuous } \\
\text { Improvement }\end{array}$ & PDCA & Kaizen Events & $\begin{array}{l}\text { Employee } \\
\text { Suggestions }\end{array}$ & $\begin{array}{c}\text { Understand } \\
\text { Systems View }\end{array}$ & $\begin{array}{l}\text { Preventative } \\
\text { Maintenance }\end{array}$ & $\begin{array}{l}\text { Supplier } \\
\text { Integration }\end{array}$ & SPC & $\begin{array}{l}\text { Technology \& } \\
\text { Equipment }\end{array}$ & - \\
\hline
\end{tabular}

Abbildung 1. Lean Warehousing Methoden [Sob09]

Die Ansätze zur Performancebewertung von Distributionszentren lassen sich neben den zugrunde gelegten Methoden nach weiteren Kriterien systematisieren. Zum einen ist eine Einteilung nach dem Untersuchungsgegenstand und zum anderen nach dem betrachteten Zeithorizont möglich. Bezüglich des Untersuchungsgegenstands lassen sich Black-Box-Ansätze und eine aufgabenbereichsspezifische Performancebewertung unterscheiden. Im Hinblick auf den betrachteten Zeithorizont lassen sich Ansätze zur operativ- und strategisch-orientierten Performancebewertung voneinander abgrenzen.

Stölzle und Gaiser stellen ein Logistik-Kennzahlensystem vor, das für einen Leistungsvergleich von Distributionslagerhäusern vorgesehen ist [SG96]. Als Grundlage des Kennzahlensystems dienen allgemeine Logistikziele. Die Autoren unterscheiden drei Analyseebenen: Gesamtlagerhaus, Lagerhausbereiche und Leistungsbereiche innerhalb der Lagerhausbereiche. Der Grad der Zielerreichung wird anhand der Zielerreichung der Leistungsbereiche des Distributionslagerhauses gemessen. Die Kennzahlen werden als Maß für die Zielerreichung definiert. Stölzle und Gaiser unterscheiden u. a. Durchsatz-, Zeit-, Qualitäts- und Komplexitätskennzahlen.

Das Distribution Center Reference Model (DCRM) wurde vom Institut für Fördertechnik und Logistiksysteme des Karlsruher Instituts für Technologie entwickelt. Das DCRM ist ein aufgabenorientiertes Instrument zum Vergleichen, Analysieren und Bewerten von Distributionszentren [FHW08]. Es erweitert die Möglichkeit des realen Benchmarkings von Distributionszentren um die Mög- lichkeit des theoretischen Benchmarkings für die Prozesse Wareneingang, Lagern und Kommissionieren, Konsolidieren und Verpacken sowie Warenausgang. Wisser entwickelte zum Zweck des theoretischen Bechmarkings analytische Berechnungsmodelle für den Prozess „Lagern und Kommissionieren“ [Wis09]. Zeit, Fläche und Kosten stellen die Performanceattribute des Systems dar. Die Performancebewertung erfolgt auf Grundlage der Topkennzahlen Kosten, Leistung und Qualität [FWS10]. Bei der Performancebewertung im DCRM wird ein Distributionszentrum nicht nur als Black Box betrachtet, es erfolgt eine detaillierte Bewertung der Aufgabenbereiche [FWS10].

McGinnis et al. veröffentlichten 2006 eine Zusammenfassung der Resultate der Benchmarking Warehouse Performance Study [MJV06]. Unter Einsatz der Data Envelopment Analysis erfolgte die Performancebewertung, bei der die Eingangsgrößen Arbeit, Investment und Fläche sowie die Ausgangsgrößen Positionsanzahl versandter Paletten, Kartons und Einzelstücke herangezogen wurden.

De Koster und Balk werten ein Benchmarking und Monitoring von Distributionszentren in Europa aus [dKB08]. Zur Performancebewertung ziehen die Autoren die Data Envelopment Analysis heran. Eingangsgrößen der Performancebewertung stellen die Anzahl direkt beschäftigter Vollzeitäquivalente, die Fläche des Distributionszentrums, den Automatisierungsgrad und die Sortimentsbreite dar. Ausgangsgrößen der Performancebewertung bilden die Anzahl kommissionierter Positionen pro Tag, das Angebot von Value Added Services, die Anzahl 
fehlerfreier Aufträge, die Bestellflexibilität sowie die Anzahl von Spezialprozessen, d. h. Prozesse, die nicht unmittelbar die operative Leistung bestimmen, sondern die längerfristige Leistung des Distributionszentrums. Die Autoren weisen auf den teilweise subjektiven Charakter der Kennzahlen hin. Außerdem arbeiten die Autoren teilweise mit Intervallangaben und linguistischen Bewertungen.

Erste Modelle zur Bewertung von Distributionszenten unter Berücksichtigung von Lean-Management-Ansätzen lassen sich in der Literatur nachweisen, z. B. von Sobanski [Sob09] oder Mahfouz [Mah11]. Beide Autoren legen den Fokus in den entwickelten Modellen auf die Bewertung des Umsetzungsstands des Lean Warehousing. Die Autoren untersuchen, welche Lean-ManagementMethoden im Distributionszentrum implementiert werden. Mahfouz schlägt verschiedene taktische und operative Lean-Kennzahlen vor: Anzahl pünktlicher Lieferungen, Anzahl stornierter Aufträge, Anteil der fertiggestellten Aufträge, Transportzeit, Anzahl fehlerfreier Aufträge, Wiederbeschaffungszeit usw. [Mah11].

Lin berücksichtigt bei der Performancebewertung von Distributionszentren die Unsicherheit der zugrunde liegenden Daten unter Verwendung der Methoden: Intervall-Valued Fuzzy Evaluation (IVFE) und Analytic Hierarchy Process (AHP) [Lin10]. In die Performancebewertung des Distributionszentrums gehen die Kennzahlen Finanzen, Lagermanagement und Kundenzufriedenheit ein.

Multi Attribute Decision Making (MADM) stellt eine Gruppe von Verfahren zur Auswahl zwischen verschiedenen Alternativen dar, die durch verschiedene Kriterien charakterisiert sind [Rib96]. Liegen die verwendeten Kriterienausprägungen oder Gewichte in Form von FuzzyZahlen vor, werden Fuzzy Multi Attribute Decision Making (FMADM)-Ansätze verwendet. In der Literatur wurden FMADM-Ansätze bereits im Kontext der Performancebewertung eingesetzt [MRP10; WL11]. Besondere Eignung besitzen FMADM-Ansätze bei Vorliegen unpräziser oder vager Daten [Rib96] und können sogar bei Vorliegen inkonsistenter Daten verwendet werden [JH03]. Die Verwendung der FMADM-Ansätze ist dann sinnvoll, wenn Ziele bzw. Kennzahlen in unscharfer Form vorliegen [MRP10].

Die Berücksichtigung von Unsicherheiten im Rahmen der Performancebewertung von Distributionszentren im Kontext des Lean Warehousing wurde nach Kenntnis der Autoren bislang in der Literatur kaum fokussiert.

\section{Methode Zur Performancebewertung}

Die Performance bezeichnet den Wertbeitrag logistischer Aktivitäten in einem Distributionszentrum und kann modellhaft an der Erfüllung der Kundenanforderungen festgemacht werden. Die Performancebewertung eines
Distributionszentrums im Kontext des Lean Warehousing orientiert sich, wie in Abschnitt 2.2 beschrieben, an den Anforderungen der Kunden. Unternehmensspezifisch werden Kundenanforderungen in individuelle Ziele überführt. In diesem Beitrag werden folgende Logistikziele nach Stölzle und Gaiser [SG96] verwendet: ,geringe Lieferzeit", „hohe Lieferzuverlässigkeit", „gute Lieferbeschaffenheit", „hohe Lieferflexibilität“" und ,geringe Logistikkosten".

Die entwickelte Methode zur Performancebewertung ermöglicht die Ermittlung der Gesamtperformance oder der Performance einzelner Bereiche eines Distributionszentrums. Die Performance eines Distributionszentrums ergibt sich durch Aggregation der Performances der verschiedenen Bereiche des Distributionszentrums: Wareneingang, Lagerbereich, Versand usw. Die bereichsspezifisch realisierten Zielausprägungen werden schrittweise zu den jeweiligen Bereichsperformances des Distributionszentrums aggregiert und anschließend in eine Gesamtperformance für das Distributionszentrum überführt.

Anschließend ist ein Kennzahlensystem zu entwickeln, in dem die Ziele durch Kennzahlen repräsentiert werden. Für die Bewertung ist es erforderlich, solche Kennzahlen heranzuziehen, die Einfluss auf die Zielerreichung und damit Kundenzufriedenheit besitzen. Die Betrachtungen beschränken sich in diesem Beitrag auf einen ausgewählten Lagerbereich. Im Kontext des Lean Warehousing ist bei der Entwicklung eines Kennzahlensystems dem Anspruch der Ressourcenorientierung Rechnung zu tragen. Die bereichsspezifische Definition der Kennzahlen erfolgt ausgehend von den im jeweiligen Lagerbereich realisierten Aufgaben. Einzelkennzahlen werden schrittweise aggregiert, um Aussagen über die Zielerreichung innerhalb des Bereichs zu ermöglichen.

Zur Aggregation der Kennzahlen werden hier zwei Methoden vorgestellt: Fuzzy Multi Attribute Decision Making (FMADM) und Fuzzy Control.

Bei der FMADM werden sowohl für die Kennzahlenwerte als auch für die Gewichtungsfaktoren FuzzyZahlen verwendet. Die Hauptmotivation liegt darin begründet, dass notwendige Daten häufig nicht exakt vorliegen oder eine Ermittlung mit hohem Aufwand verbunden ist.

Zur Kennzahlaggregation werden Rechenvorschriften für triangulare Fuzzy-Zahlen mit den Zugehörigkeitsfunktionen $\mu\left(\mathrm{x}_{1}\right)=\left(\mathrm{l}_{1}, \mathrm{~m}_{1}, \mathrm{u}_{1}\right)$ und $\mu\left(\mathrm{x}_{2}\right)=\left(\mathrm{l}_{2}, \mathrm{~m}_{2}, \mathrm{u}_{2}\right)$ verwendet, die von Dubois und Prade [DP80] vorgestellt wurden (vgl. auch [Rib96]).

$$
\begin{aligned}
& \left(\mathrm{l}_{1}, \mathrm{~m}_{1}, \mathrm{u}_{1}\right) \oplus\left(\mathrm{l}_{2}, \mathrm{~m}_{2}, \mathrm{u}_{2}\right)=\left(\mathrm{l}_{1}+\mathrm{l}_{2}, \mathrm{~m}_{1}+\mathrm{m}_{2}, \mathrm{u}_{1}+\mathrm{u}_{2}\right) \\
& \left(\mathrm{l}_{1}, \mathrm{~m}_{1}, \mathrm{u}_{1}\right) \otimes\left(\mathrm{l}_{2}, \mathrm{~m}_{2}, \mathrm{u}_{2}\right)=\left(\mathrm{l}_{1} \times \mathrm{l}_{2}, \mathrm{~m}_{1} \times \mathrm{m}_{2}, \mathrm{u}_{1} \times \mathrm{u}_{2}\right) \\
& \left(\mathrm{l}_{1}, \mathrm{~m}_{1}, \mathrm{u}_{1}\right) \div\left(\mathrm{l}_{2}, \mathrm{~m}_{2}, \mathrm{u}_{2}\right)=\left(\mathrm{l}_{1} \div \mathrm{l}_{2}, \mathrm{~m}_{1} \div \mathrm{m}_{2}, \mathrm{u}_{1} \div \mathrm{u}_{2}\right)
\end{aligned}
$$


Eine Aggregation der Kennzahlen kann durch Bestimmung des gewichteten Mittelwerts vorgenommen werden. Der gewichtete Mittelwert R ergibt sich als Quotient aus der Summe der Produkte der Performancebeiträge $\tilde{p}_{n}$ (Performancebeitrag des Werts einer Kennzahl n) mit den Gewichtungsfaktoren $\widetilde{\mathrm{w}}_{\mathrm{n}}$ der Kennzahlen und der Summe der Gewichtungsfaktoren. Der gewichtete Mittelwert kann wie folgt bestimmt werden:

$\mathrm{R}=\left(\begin{array}{llll}\tilde{\mathrm{p}}_{11} & \tilde{\mathrm{p}}_{12} & \cdots & \tilde{\mathrm{p}}_{1 \mathrm{n}}\end{array}\right) \otimes\left(\begin{array}{c}\widetilde{\mathrm{w}}_{1} \\ \vdots \\ \widetilde{\mathrm{w}}_{\mathrm{n}}\end{array}\right) \div \sum_{\mathrm{i}=1}^{\mathrm{n}} \widetilde{\mathrm{w}}_{\mathrm{i}}$

Die strenge Ausrichtung der Kennzahlen an den Logistikzielen ermöglicht die Ermittlung des Wertbeitrags der verschiedenen Bereiche des Distributionszentrums. Die Kennzahl R kann somit als Performanceindikator herangezogen werden. Diese Kennzahl kann auf Bereichsebene sowie für das gesamte Distributionszentrum ermittelt werden.

Alternativ zum FMADM-Ansatz erscheint der Einsatz der Methode Fuzzy Control geeignet. Dieser Ansatz bietet die Möglichkeit, die Risikoaversion verschiedener Entscheidungsträger zu berücksichtigen. Die Definition der Regelbasis ist im Rahmen des Knowledge Engineerings durch Experten durchzuführen. Im Rahmen dieser Methode sind mehrere Regelbasen zu erstellen. Zum einen müssen Regeln zur Aggregation der Bereichsziele und zum anderen zur Aggregation der Bereichsperformances definiert werden.
Die entwickelte Methode für die Performancebewertung wird folgend an einem Fallbeispiel vorgestellt.

\section{EIN FALLBEISPIEL}

Die erläuterte Methode wurde am Beispiel eines Distributionszentrums des Lebensmitteleinzelhandels erarbeitet. Die Betrachtungen konzentrieren sich auf den Bereich des Wareneingangs. Im Fallbeispiel umfasst der Begriff „Wareneingang“ alle Prozesse bis zur Auslagerung der Artikel in die Greifzonen für die Kommissionierung, d. h. Warenannahme, Identifikation, Wareneinlagerung und Warenauslagerung in die Greifzonen.

Des Weiteren wurden folgende Logistikziele zugrunde gelegt: kurze Lieferzeiten, hohe Lieferzuverlässigkeit, hohe Lieferflexibilität, gute Lieferbeschaffenheit sowie geringe Logistikkosten. Die Logistikziele wurden schrittweise konkretisiert. Dies erlaubt die Ableitung von Kennzahlen( vgl. Abbildung 2).

Die Logistikkosten werden durch die in Anspruch genommenen Flächen, das Personal und anteilige Investitionskosten für die Logistikimmobilie sowie Förder- und Lagertechnik bestimmt. Die Betrachtungen beschränken sich hier auf die Personalkosten. Die Logistikkosten werden durch die Kennzahl „Anzahl der Mitarbeiter im Wareneingang" charakterisiert.

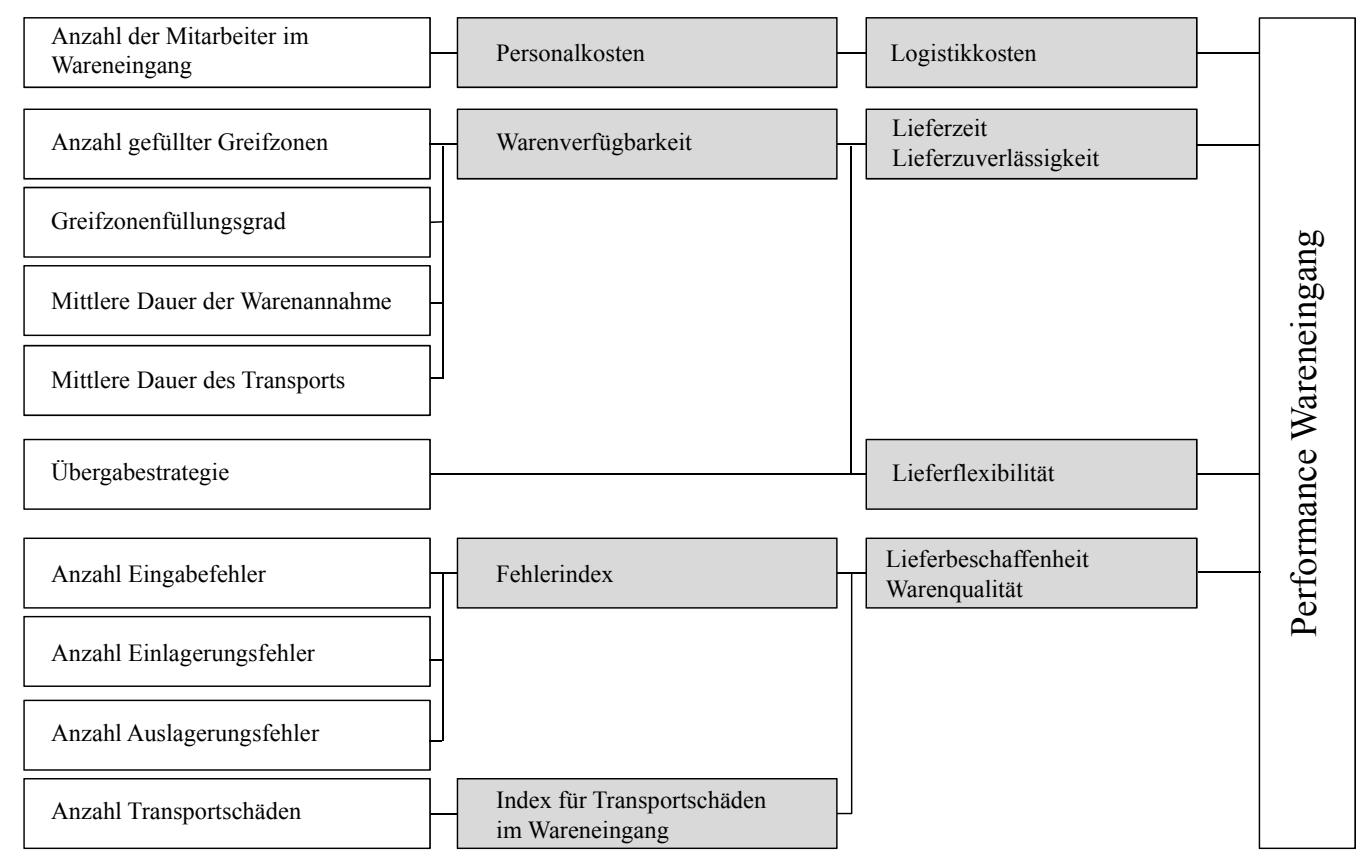

Abbildung 2. Kennzahlensystem nach Zielsystematik

Die Lieferzeit und -zuverlässigkeit werden durch die Kennzahl „Warenverfügbarkeit“ bestimmt. Für die Kennzahl „Warenverfügbarkeit“" wurden die Kennzahlen „Anzahl gefüllter Greifzonen“, „Greifzonenfüllungsgrad“, „mittlere Dauer der Warenannahme“ und „mittlere Dauer des Transports" gewählt. Die Waren werden von den Zählkräften nach Anlieferung in Empfang genommen und nach Erfassung und Kontrolle durch Staplerfahrer an die 
zugehörigen Lagerplätze verbracht. Sind Greifzonen nicht gefüllt, kann ein Kommissionierauftrag nicht komplettiert werden.

Das Logistikziel „hohe Lieferflexibilität“ wird durch die Warenverfügbarkeit und die Übergabestrategie im Wareneingang beeinflusst. Die Übergabestrategie im Wareneingang steht in unmittelbarem Zusammenhang mit der Möglichkeit, leere Greifzonen rechtzeitig zu füllen. Denkbar sind sowohl eine Zug-um-Zug-Verräumung der Waren im Wareneingang, eine Komplettbearbeitung eines Wareneingangsauftrags, eine priorisierte Verräumung bestimmter Artikel als auch eine priorisierte Verräumung eines ganzen Auftrags.

Das Ziel „hohe Lieferbeschaffenheit“ bzw. „hohe Warenqualität" wird durch die Ziele „Vermeidung von Fehlern“ sowie „Vermeidung von Transportschäden im Wareneingang" konkretisiert. Die Vermeidung von Fehlern wird durch die Kennzahl „Fehlerindex“ repräsentiert, die wiederum durch die Kennzahlen „Anzahl Eingabefehler“ (z. B. falsche MHD-Eingaben), „Anzahl Einlagerungsfehler“ (z. B. falscher Lagerort) und „Anzahl Auslagerungsfehler" (z. B. fehlerhafte Reihenfolge bei der Auslagerung in die Greifzonen in Bezug auf das MHD) determiniert wird. Die Vermeidung innerbetrieblicher Transportschäden wird durch die Kennzahl „Index für Transportschäden im Wareneingang“ bzw. „Anzahl Transportschäden“" bestimmt.

Mit Hilfe dieses Kennzahlensystems kann die Performance des Wareneingangsbereichs ermittelt werden. Zunächst wird der FMADM-Ansatz verwendet, anschließend die Methode Fuzzy Control.

\section{FMADM-Ansatz zur Performancebewertung}

Bei Verwendung des FMADM-Ansatzes sind zunächst der Performancebeitrag der ermittelten Kennzahlenwerte und die Gewichtung der Kennzahlen zu ermitteln. Dazu erfolgt vorbereitend eine Modellierung der Fuzzy-Mengen für die linguistischen Variablen „Gewichtung“ und „Performancebeitrag“. Zur Modellierung der Gewichtung der Kennzahlen wurden die linguistischen Terme (,gering“, „mittel“, „hoch“) auf einer Skala von 0 bis 1 abgebildet. Zur Modellierung des Performancebeitrags wurde eine Partition mit fünf Fuzzy-Mengen gewählt, die die linguistischen Terme „sehr gering“, ,gering“, „mittel“, „hoch“ und „sehr hoch“ abbildet. Abbildung 3 zeigt die Fuzzy-Mengen für den Performancebeitrag und die Gewichtung. Zur Vereinfachung wurden ausschließlich triangulare Fuzzy-Mengen verwendet. Eine Erweiterung auf beliebige Formen der $\mathrm{Zu}$ gehörigkeitsformen ist möglich. Obige Rechenvorschriften sind entsprechend zu modifizieren. Dubois und Prade stellen dafür einen Algorithmus bereit [DP80].

Anschließend erfolgt eine Ermittlung der Gewichtung der Kennzahlen im Hinblick auf die Performance des Wa- reneingangs. Durch Experten wird jeder Kennzahl eine Gewichtung in Form eines linguistischen Terms zugeordnet.

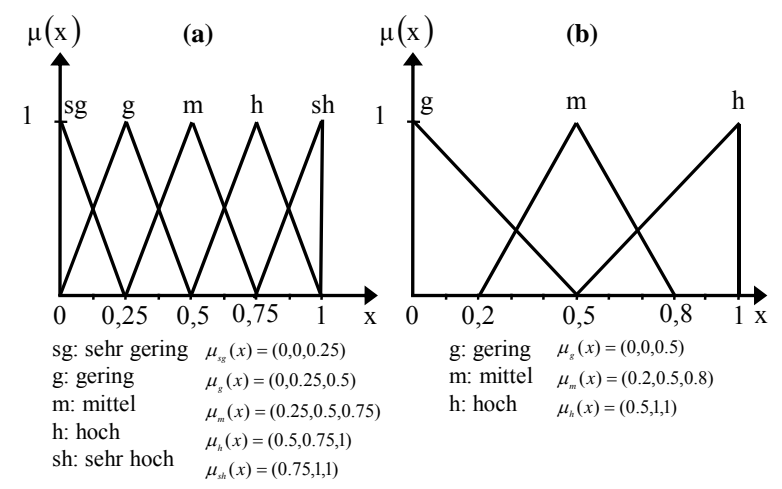

Abbildung 3. Fuzzy-Mengen für den Performancebeitrag (a) und die Gewichtung (b)

Weiterhin sind die Kennzahlenwerte im Bereich des Wareneingangs zu ermitteln. Durch Experten werden den Kennzahlenwerten linguistische Terme zugeordnet, die den Performancebeitrag charakterisieren. Scharfe und unscharfe Kennzahlwerte werden so in linguistische Terme überführt. Abbildung 4 zeigt die Kennzahlenwerte sowie die durch die Experten festgelegten Gewichtungen der Kennzahlen und die zugeordneten Performancebeiträge.

\begin{tabular}{|c|c|c|c|}
\hline Kennzahlen & Bedeutung & $\begin{array}{c}\begin{array}{c}\text { Kennzahl- } \\
\text { wert }\end{array} \\
\end{array}$ & \begin{tabular}{|c}
$\begin{array}{c}\text { Performance- } \\
\text { beitrag }\end{array}$ \\
\end{tabular} \\
\hline Logistikkosten & hoch & & \\
\hline Anzahl der Mitarbeiter im Wareneingang & hoch & 8 & mittel \\
\hline Lieferzeit & mittel & & \\
\hline Warenverfügbarkeit & hoch & & \\
\hline Anzahl gefüllter Greifzonen & mittel & 95 & sehr hoch \\
\hline Greifzonenfüllungsgrad & mittel & $80 \%$ & hoch \\
\hline Mittlere Dauer der Warenannahme & hoch & $80 \mathrm{~s}$ & hoch \\
\hline Mittlere Dauer des Transports & hoch & $210 \mathrm{~s}$ & mittel \\
\hline Lieferflexibilität & gering & & \\
\hline Warenverfügbarkeit & hoch & & \\
\hline Übergabestrategie & gering & Zug-um-Zug & mittel \\
\hline Lieferbeschaffenheit & mittel & & \\
\hline Fehlerindex & gering & & \\
\hline Anzahl Eingabefehler & hoch & 10 & gering \\
\hline Anzahl Einlagerungsfehler & hoch & 5 & mittel \\
\hline Anzahl Auslagerungsfehler & mittel & 3 & hoch \\
\hline Anzahl Transportschäden & gering & 5 & gering \\
\hline
\end{tabular}

Abbildung 4. Gewichtung, Kennzahlenwert und Performancebeitrag der Kennzahlen

Mit Hilfe der fuzzifizierten Performancebeiträge der Kennzahlen und der Kennzahlgewichte erfolgt nun die fuzzybasierte Kennzahlenaggregation unter Verwendung des gewichteten Mittels. Die den Logistikzielen zugeordneten Kennzahlen sowie die Performance des Wareneingangsbereichs können entsprechend obigen Gleichungen ermittelt werden:

$$
\mathrm{R}_{\text {Logistikkosten }}=(0.25,0.5,0.75) \text {, }
$$$$
\mathrm{R}_{\text {Lieferzeit }}=(0.46,0.71,0.93) \text {, }
$$ 
$\mathrm{R}_{\text {Lieferflexibilität }}=(0.46,0.71,0.88)$,

$\mathrm{R}_{\text {Lieferbeschaffenheit }}=(0.1,0.35,0.61)$,

$\mathrm{R}_{\text {Wareneingang }}=(0.26,0.51,0.78)$.

Die ermittelten Kennzahlen liegen als Fuzzy-Zahlen vor und sind abschließend in scharfe Zahlen zu überführen. Dazu können verschiedene Defuzzifizierungsverfahren eingesetzt werden. Besonders häufig wird die Flächenschwerpunktmethode verwendet. Für triangulare $\mathrm{Zu}$ gehörigkeitsfunktionen $\mu(x)=(a, b, c)$ können die scharfen Werte wie folgt ermittelt werden.

$\mathrm{d}(\mu(\mathrm{x}))=(\mathrm{a}+\mathrm{b}+\mathrm{c}) / 3$

Es resultiert für die Performance des Wareneingang ein scharfer Wert von $\mathrm{d}\left(\mathrm{R}_{\text {Wareneingang }}\right) \approx 52 \%$.

\section{Fuzzy Control zur Performancebewertung}

Der Einsatz der Methode Fuzzy Control im Rahmen der Performancebewertung bietet sich an, wenn eine analytische Bestimmung der Performance nicht möglich oder nur mit großem Aufwand realisierbar ist. Außerdem ist die Verwendung sinnvoll, wenn Expertenwissen von Bedeutung ist, z. B. bei interdependenten Zielen.

Ein Fuzzy-Controller besteht aus den Komponenten Fuzzifizierung, unscharfe Inferenz und Defuzzifizierung. Scharfe Eingangswerte werden im Rahmen der Fuzzifizierung in Fuzzy-Zahlen überführt. Unter Verwendung der Regelbasis - bestehend aus linguistischen Regeln (WennDann-Regeln) - erfolgt eine unscharfe Inferenz. Sie legt die Zusammenhänge zwischen den Eingangsgrößen und den Ausgangsgrößen des Systems fest. In der InferenzMaschine werden Fuzzy-Ausgangsgrößen erzeugt, die bei der Defuzzifizierung in scharfe Größen überführt werden.

Nach Ableitung eines Kennzahlensystems sind bei der Performancebewertung mittels Fuzzy Control zunächst die Kennzahlen als Fuzzy-Mengen zu modellieren. Im Unterschied zum FMADM-Ansatz wird nicht der Performancebeitrag eines Kennzahlenwerts abgebildet, sondern die Kennzahl selbst. Damit können für jede Kennzahl differenzierte Fuzzy-Mengen gebildet werden. Die Entwicklung der Fuzzy-Mengen für die verschiedenen Kennzahlen erfolgt in Zusammenarbeit mit Experten. Beispielsweise können für die Kennzahl „Mittlere Dauer der Warenannahme“ die linguistischen Terme „sehr kurz“, „,kurz“, „mittel“, „lang“ und „sehr lang“ definiert werden. Der scharfen, gemessenen mittleren Dauer der Warenannahme von z. B. 84 Sekunden werden, wie in Abbildung 5 ersichtlich, die linguistischen Terme „mittel“ und „lang“" zugeordnet, jedoch mit unterschiedlich ausgeprägter Zugehörigkeit $(0,6$ bzw. 0,4). Neben der Verwendung scharfer Kennzahlenwerte erlaubt die Verwendung von Fuzzy Control ebenfalls die Arbeit mit unscharfen Eingangsgrößen.
Die Performance des Wareneingangs ergibt sich durch Anwendung von Wenn-Dann-Regeln, die von Experten zu entwickeln sind. Die Regeln modellieren die Abhängigkeit der Performance von verschiedenen Kennzahlen. Nach Überführung der scharfen Kennzahlenwerte in Fuzzy-Mengen, werden die fuzzifizierten Kennzahlenwerte durch Regeln verknüpft. Ein Beispiel für eine Regel ist: Wenn $\left(\right.$ Logistikkosten $=$, gering") ${ }^{\prime \prime}$ und $($ Lieferzeit $=$ „gering") und (Lieferflexibilität = ,hoch") und (Lieferbeschaffenheit $=$,ausgezeichnet"), dann Wareneingangsperformance $=$,gut ${ }^{\text {“ }}$.

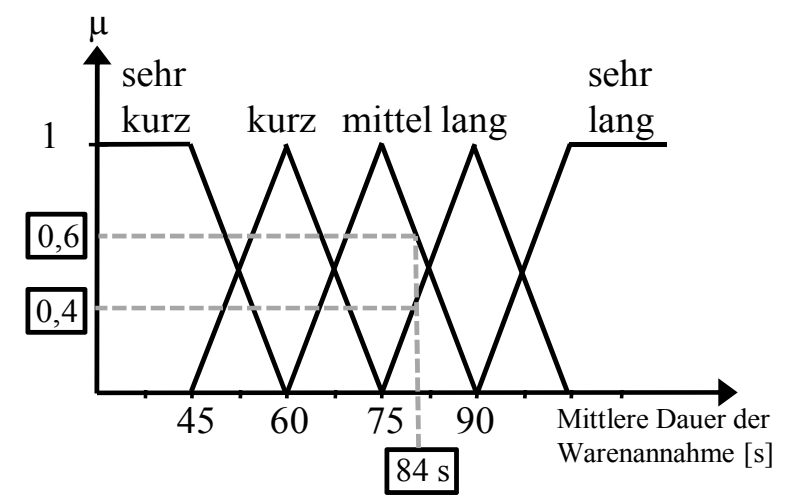

Abbildung 5. Graduelle Zugehörigkeit am Beispiel der Kennzahl "Mittlere Dauer der Warenannahme"

Die Kennzahlen werden stufenweise regelbasiert zur Performance des Wareneingangs zusammengeführt. Hierzu kann die Software Matlab (Version R2011b) eingesetzt werden. Das Programm besitzt eine integrierte FuzzyLogic-Toolbox. Abbildung 6 veranschaulicht die Zusammenführung der Kennzahlen in Matlab. Die Kennzahlen „Anzahl gefüllter Greifzonen“, „Greifzonenfüllungsgrad“, „Mittlere Dauer der Warenannahme“ und „Mittlere Dauer des Transports" werden im Regler zusammengeführt. Ausgabewert ist die fuzzifizierte Warenverfügbarkeit.

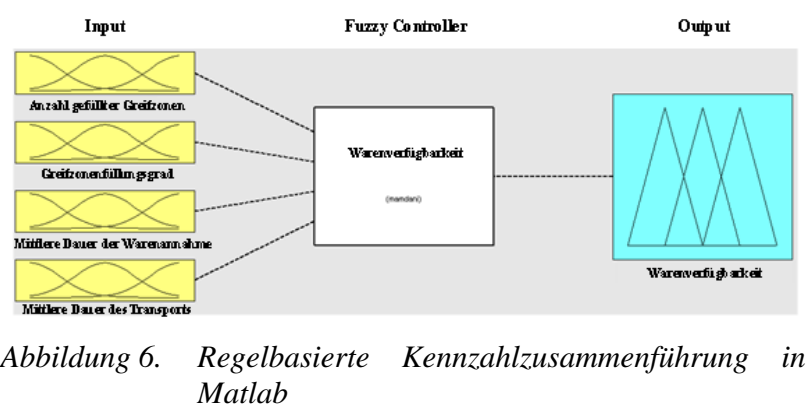

Abbildung 7 zeigt exemplarische Regeln für obiges Beispiel. Die scharfen Kennzahlenwerte wurden den zugehörigen Fuzzy-Mengen zugeordnet. Die aus den Regeln resultierenden Warenverfügbarkeiten wurden anschließend zusammengeführt und defuzzifiziert. Es ergibt sich für die Warenverfügbarkeit ein defuzzifizierter Wert von 37,5. Dieser Wert geht anschließend in andere Regeln ein, z. B. in die Regeln zur Bestimmung der Lieferzeit oder Lieferflexibilität. Die Bestimmung der Performance des Wareneingangs erfolgt entsprechend. 


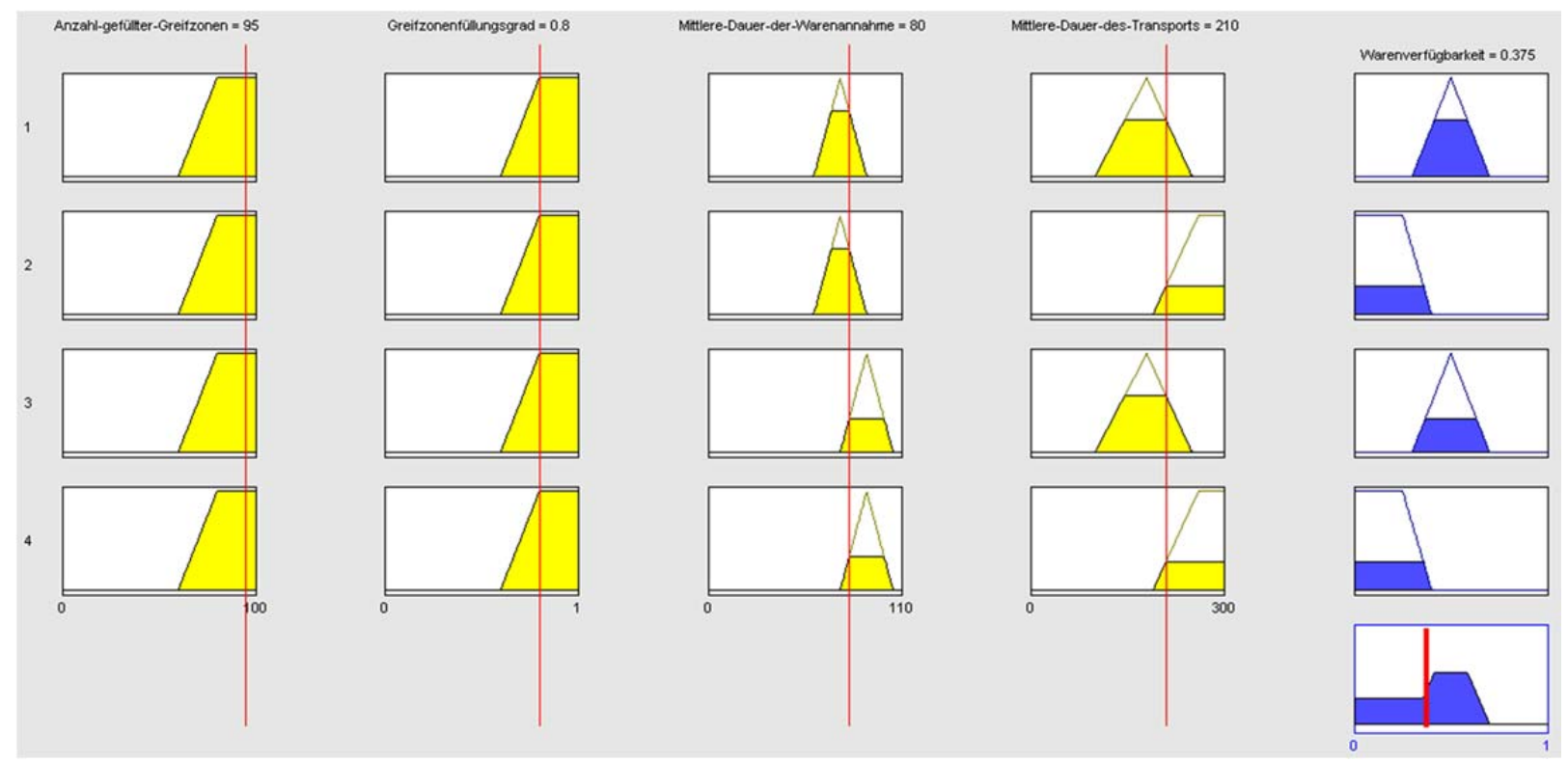

Abbildung 7. Auszug aus einer Regelbasis für die Warenverfügbarkeit

\section{FAzIT UND AUSBLICK}

Eine effiziente Prozess- und Systemgestaltung erfordert eine kontinuierliche Bewertung der entsprechenden Performance. Ziel des Beitrags war es, eine Methode für die Performancebewertung von Distributionszentren vorzustellen, die den Anforderungen des Lean Warehousing gerecht wird. Die Betrachtungen konzentrierten sich dabei auf den Bereich des Wareneingangs. Die vorgestellte Methodik ist auf andere Bereiche eines Distributionszentrums übertragbar. Die bereichsspezifischen Kennzahlensysteme sind dahingehend zu modifizieren. Es ist davon auszugehen, dass die Bedeutung unzureichend quantifizierbarer Ziele, unscharfer Kennzahlwerte oder menschliches Fachwissen über bestimmte Anwendungsdomänen eine immer größere Bedeutung erlangen wird. Im Beitrag wurde deshalb eine Methode entwickelt, die die Berücksichtigung unscharfer Daten ermöglicht. Die im Rahmen der Methode erforderliche Kennzahlenaggregation kann mit verschiedenen Verfahren realisiert werden. Exemplarisch wurden zwei fuzzybasierte Verfahren vorgestellt. Perspektivisch wird eine Verfeinerung des zugrunde gelegten Kennzahlensystems angestrebt. Außerdem sollen die Möglichkeiten der Modellierung qualitativer Ziele, wie z. B. die ökologieorientierte Gestaltung von Distributionszentren, weiter untersucht werden. Eine Vertiefung des Dialogs mit Praxispartnern ist vorgesehen.

\section{LITERATUR}

[ALMS08] Alicke, K.; Leopoldseder, M.; Mishra, D.; Schulze, W.-A.: What's in your warehouse?. McKinsey\&Company, http://www.mckinsey.it/storage/first/upl oad-
[Chu09] Chua, C. W.; Katayama, H.: Lean Approaches in Warehousing Design and Management for Automotive Parts Supply Operation. In: International Conference Asia Pasific Industrial Engineering \& Management Systems Conference (APIEM) 2009, Kitakyushu, S. 338-349

[DP80] Dubois, D.; Prade, H.: Fuzzy Sets and Systems: Theory and Application. New York: Academic Press, 1980

[dKB08] de Koster, R.; Balk, B. M.: Benchmarking and Monitoring International Warehouse Operations in Europe. In: Production and Operations Management, 17, 2008, S. 175-183

[FHW08] Furmans, K.; Huber, C.; Wisser, J.: Leistungsfähigkeit von Distributionszentren systematisch vergleichen. In: $\mathrm{f}+\mathrm{h}$ Fördern und Heben, 1/2, 2008, S. $15-17$

[FWS10] Furmans, K.; Wisser, J.; Schwab, M.: Kosten- und Leistungsstrukturen in Distributionszentren. Aktuelle Ergebnisse der Warehouse-Excellence-Studie. In: $\mathrm{f}+\mathrm{h}$ Fördern und Heben, 7/8, 2010, S. 257-259

[Gla08] Glass, M.: Schnellläuferstrategien in Lagern und Dynamische Zonierung. Dissertation, TU Chemnitz, 2008 
[GM05] Goldsby, T.; Martichenko, R.: Lean Six Sigma. Strategic Development to Operational Success. Boca Raton: J. Ross Publishing, 2005

[Hel08] Hellingrath, B.: Planung und Bewertung von Produktionsprozessen. In: Arnold, D. (Hrsg.) et. al: Handbuch Logistik. 3. Auflage, Berlin, Heidelberg: Springer-Verlag, 2008, S. 449-456

[JH03] Jiang, B. C.; Hsu, C.-H.: Development of a fuzzy decision model for manufacturability evaluation. In: Journal of Intelligent Manufacturing, 14, 2003, S. 169-181

[JHR97] Jones, D. T.; Hines, P.; Rich, N.: Lean Logistics. In: International Journal of Physical Distribution \& Logistics, 27(3/4), 1997, S. 153-173

[Küh05] Kühner, M.: Ein Verfahren zur Analyse prozessualer Logistikleistung auf Basis der Data Envelopment Analysis, Dissertation, Universität Stuttgart, 2005

[Lee97] Lee, H.F.: Performance analysis for automated storage and retrieval systems. In: IIE Transactions, 29(1), 1997, S. $15-28$

[Lin10] Lin, C.: Management Performance Evaluating in Distribution Center. In: Logistics and Supply Chain Research in China - Proceedings of The 3rd International Conference on Logistics and Supply Chain Management 2010, Hunan, 2010, S. 173-179

[Mah11] Mahfouz, A.: An Integrated Framework to Assess 'Leanness' Performance in Distribution Centres, Dissertation, Dublin Institute of Technology, 2011

[MJV06] McGinnis, L. F.; Johnson, A.; Villarreal, M.: Benchmarking Warehouse Performance Study. 2006

[MRP10] Mohanty, R.; Ravi, V.; Patra, M. R.: Application of Fuzzy Multi Attribute Decision Making Analysis to Rank Web Services. In: International Conference on Computer Information Systems and Industrial Management Applications (CISIM), 2010, S. 398-403

[Rib96] Ribeiro, R. A.: Fuzzy multiple attribute decision making: A review and new preference elicitation techniques. In: Fuzzy Sets and Systems, 78, 1996, S. $155-181$

[SB08] Stölzle, W.; Bachmann, H.: Performance Management in der Logistik. In: Arnold, D. (Hrsg.) et. al: Handbuch Logistik. 3. Auflage, Berlin, Heidelberg: Springer-Verlag, 2008, S. 917-927

[See06] Seemüller, S.: Durchsatzberechnung automatischer Kleinteilelager im Umfeld des elektronischen Handels, Dissertation, TU München, 2006

[SG96] Stölzle, W.; Gaiser, C.: LogistikKennzahlensysteme. Kennzahlen als Instrument für den Leistungsvergleich von Distributionslagerhäusern. In: Controlling, 8, 1996, S. 40-48

[Sob09] Sobanski, E.B.: Assessing Lean Warehousing: Development and Validation of a Lean Assessment Tool. Dissertation, Oklahoma State University, 2009

[Stö02] Stölzle, W.: Supply Chain Controlling and Performance Management - Konzeptionelle Herausforderungen für das Supply Chain Management. In: Logistik Management, 4, 2002, S. 10-21

[Vas08] Vastag, A.: Beschreibung und Abgrenzung der Distribution. In: Arnold, D. (Hrsg.) et. al: Handbuch Logistik. 3. Auflage, Berlin, Heidelberg, 2008, S. 405-412

[Wis09] Wisser, J.: Der Prozess Lagern und Kommissionieren im Rahmen des Distribution Center Reference Model (DCRM). Dissertation, Universität Karlsruhe (TH), 2009

[WJ03] Womack, J. P.; Jones, D. T.: Lean Thinking. Banish Waste and Create Wealth in Your Corporation. New York: Free Press, 2003

[WL11] Wang, S.-Y.; Lee, C.-F.: Fuzzy multicriteria decision-making for evaluating mutual fund strategies, In: Applied Economics, 43, 2011, S. 3405-3414

[Zah65] Zadeh, L.A.: Fuzzy sets. In: Information and Control, 8 (3), 1965, S. 338-353 